\title{
Formulation, Optimization and Evaluation of in-situ Gelling Liquid Oral Formulation of a Novel Antidiabetic Drug: Canagliflozin
}

\author{
Rohith Ganapathi Bhatta, Sadashivaiah Rudragangaiah, Satheesha Babu Birur Kotappa* \\ Department of Pharmaceutics, Government College of Pharmacy, \#2, P Kalingarao road, Subbaiah Circle, Bangalore, Karnataka, \\ INDIA.
}

\begin{abstract}
Aim: The present research work involves the development and optimization of an in-situ gel forming formulation by face centered central composite response surface design methodology to increase buoyancy and controlled release of a novel antidiabetic drug; Canagliflozin hemihydrate, having absorption window in upper part of gastrointestinal tract. Materials and Methods: The drug and excipient compatibility studies were performed by subjecting to Fourier Transform Infra-red spectroscopy and Differential Scanning Calorimetry analysis. Nine primary formulations suggested by Design Expert software were formulated and subjected for the evaluation by measurement of rheological properties, buoyancy studies, drug content and in-vitro drug release studies. The optimization method adopted in the present study was face centered central composite response surface design. Result: The effect of two independent factors namely concentration of sodium alginate and concentration of gas generating agent calcium carbonate on responses like floating lag time, viscosity and in-vitro drug release were considered for the optimization by plotting three dimensional surface response plots. The results of these studies were subjected to analysis by software to obtain an optimized formulation. The software suggested the optimized formulation must consists of $0.868 \%$ of sodium alginate and $0.957 \%$ of calcium carbonate and these compositions are much near to the composition of SF5. Conclusion: By considering the effect of calcium cations on the gelling properties of various concentration of sodium alginate, it was concluded that the effective gelling was achieved in the formulation comprised of $1 \%$ sodium alginate with $0.5 \%, 1.0 \%$ and $1.5 \%$ of calcium carbonate.

Key words: Gastric retention, Antidiabetic, Canagliflozin Hemihydrate, Sodium Alginate, Optimization.
\end{abstract}

\section{INTRODUCTION}

Short Gastric Residence Time (GRT) and unpredictable Gastric Emptying Time (GET) are the physiological adversities challenge the development of rate controlled oral drug delivery systems. To overcome these, certain scientific and technological advancements have been made. ${ }^{1}$ It is always better to develop ideal drug delivery systems that will be a single dose for the duration of treatment and deliver the drug directly at the site of action.

The bioavailability of drugs with narrow absorption window in stomach (like captopril, metformin, riboflavin etc.) can be increased thus reducing the dose. The stomach with a highly acidic $\mathrm{pH}$ is not free from microbial colonization like of Helicobacter pylori ${ }^{2,3}$ (leads to ulcer) or other organisms. By targeting drugs like amoxicillin, metronidazole, ${ }^{3}$ tetracycline ${ }^{3}$ and anti-histamines help in eradication of pathogenic organisms and avoid dose dumping.

Gastro-retentive Drug Delivery System (GRDDS) has gained immense popularity in the field of oral drug delivery recently. It is a widely employed approach to retain the dosage form in the stomach for an extended period of time and release the
Submission Date: 26-03-2019; Revision Date: 10-04-2019; Accepted Date: 22-04-2019.

DOI: 10.5530/ijper.53.2s.56 Correspondence:

Dr. Satheesha Babu Birur Kotappa

Associate Professor, Department of Pharmaceutics,

Government College of Pharmacy, \#2, P Kalingarao Road, Bangalore- 560027, Karnataka, INDIA.

Phone no: +919481687878

Email: bksathishbabu@ gmail.com 
drug slowly that can address many challenges associated with conventional oral delivery, including poor bioavailability. Different innovative approaches like magnetic field assisted gastro-retention, plug type swelling system, muco-adhesion technique, floating system with or without effervescence are being applied to fabricate GRDDS.

Conventional oral dosage forms pose low bioavailability problems due to their rapid gastric transition from stomach. Drugs which produce their local action in stomach get rapidly emptied and do not get enough residence time in stomach. So, frequency of dose administration in such cases is increased. To avoid this problem, various efforts have been made to prolong the retention time of drug delivery system.

Several approaches are currently utilized in the prolongation of the GRT, including Floating Drug Delivery Systems (FDDS), are also known as Hydro Dynamically Balanced Systems (HBS), swelling and expanding systems, polymeric bio-adhesive systems, ${ }^{4}$ modified shape systems and high-density systems. The various buoyant preparations include hollow microspheres ${ }^{5,6}$ (micro balloons), granules, powders, capsules, tablets and laminated films.

Sodium alginate is widely used in pharmaceutical formulations in beads, matrix tablets and microcapsules. In presence of di- and trivalent metal ions, dilute solutions of sodium alginate undergoes gelation by a process involving consecutive guluronic residues in the guluronic acid $(G)$ blocks of the alginate chain. This property is successfully utilized by many researchers for the formulation of novel drug delivery systems. ${ }^{7}$ Canagliflozin hemihydrate is the recently FDA approved first oral agent in a novel class of diabetes drugs known as sodium-glucose co-transporter-2 (SGLT-2) inhibitors. Canagliflozin is rapidly absorbed in the gastrointestinal (GI) tract. It has a relative oral bioavailability of $65 \%$. It can be taken without regard to food, but it is recommended that it be taken before the first meal of the day. ${ }^{8}$ Solubility of Canagliflozin is of major concern as it is insoluble in water. With all these properties of the Canagliflozin, in this work we have tried to develop a dosage form which can address and overcome the challenges in achieving efficient drug delivery.

\section{MATERIALS AND METHODS}

\section{Materials}

Canagliflozin was purchased from Watson Pharma Private Limited, Mumbai, India, Sodium alginate was a gift sample from Anthem Bio Sciences Private Limited, Bengaluru, India and all other chemicals and reagents used were of research grade.

\section{Methods}

\section{Drug identification and authentication}

Canagliflozin identified and authenticated by determining melting point (Lab India, Visual Melting Range Apparatus, MR-VIS'), solubility, Fourier-Transform Infrared spectroscopy (FTIR) (Shimadzu IR Affinity$1 \mathrm{~S}$ instrument) and Differential Scanning Calorimetry (DSC) (Mettler star SW-9.01) spectral analysis.

\section{Drug polymer compatibility studies}

Polymer and drug Canagliflozin compatibility study was carried out by subjecting the drug and polymer (sodium alginate) individually as well as in physical mixture into FTIR and DSC.

\section{Fourier transform infrared spectroscopy}

FTIR studies were carried out in Shimadzu IR Affinity-1S instrument. The individual polymer and pure drug were placed on the germanium prism and FTIR spectra were recorded in the scanning measurement range of 600 to $4000 \mathrm{~cm}^{-1}$. In the same manner FTIR spectra were recorded for the physical mixtures of polymer and drug (1:1 ratio).

\section{Differential scanning calorimetry}

DSC studies were carried out in Mettler star SW-9.01 instrument. About 1-3 mg of polymer, pure drug and the physical mixtures of polymer and drug (1:1 ratio) were placed in aluminum pans and the pans were closed by crimping the lids. The filled aluminum pans and empty sealed pan were placed in a sample holder where empty pan acts as a reference. The thermal behavior of samples was investigated under nitrogen purging at scanning rate of $10^{\circ} \mathrm{C} / \mathrm{m}$, covering the temperature range of $30-360^{\circ} \mathrm{C}$. Heat runs for each sample were set from $30-360^{\circ} \mathrm{C}$ using nitrogen as purging gas and the samples were analysed.

\section{Optimization of floating in-situ gelling formulation using Design Expert software}

A two-level three-response surface face centered central composite design for experimentation is utilized in present study. Statistical experimental design was performed using DESIGN EXPERT ${ }^{\circledR}$ version 11.0 (Stat-Ease Inc., Minneapolis, USA) software. Response surface graphs were used to show the factor interaction between the considered variables. Selected independent variables studied were the concentration of sodium alginate (X1); and Calcium carbonate (X2) added to the formulation. Three factorial levels coded for low, mediumand high settings $(-1,0$ and +1 , respectively) were considered for two independent variables. The selected dependent variables investigated were the viscosity (Y1), floating lag time (Y2), 
percentage of drug released at $1^{\text {st }} \mathrm{h}(\mathrm{Y} 3)$ and $8^{\text {th }} \mathrm{h}(\mathrm{Y} 4)$. A total of 9 (Table 4) experimental runs were required for analyzing the interaction of each level on formulation characters and to optimize. Results obtained for different evaluation parameters were subjected to software for analysis and further optimization of the formulations. ${ }^{9-12}$

\section{Formulation of gastro retentive in-situ gelling liquid}

Sodium alginate, at solution concentrations of $0.5-$ $1.5 \%(\mathrm{w} / \mathrm{v})$ were prepared in de-ionized water by heating to $60^{\circ} \mathrm{C}$ with continuous stirring. After cooling below $40^{\circ} \mathrm{C}$, various concentrations of calcium carbonate and drug solutions were added and dispersed well with continuous stirring. The resulting sodium alginate in-situ gel solution containing Canagliflozin was finally stored in amber color narrow mouth bottles until further use. ${ }^{13}$ (Table 1 )

\section{Evaluation}

\section{Rheological properties of the formulations}

The viscosities of formulations were determined by Brookfield viscometer DV-III using spindle no. 21. Viscosity was measured at different Rotations Per Min (RPM) to get corresponding angular velocities and the findings are depicted in Table $2 .{ }^{13}$

\section{Drug content}

One $\mathrm{ml}$ of the formulation added to $10 \mathrm{ml}$ volumetric flask with $7.5 \mathrm{ml}$ of methanol and stirred for 1 $\mathrm{h}$ on magnetic stirrer and made up to the mark with methanol. The solution was filtered and $1 \mathrm{ml}$ of this solution is transferred to $10 \mathrm{ml}$ volumetric flask and made up the mark with mobile phase. The amount of Canagliflozin was estimated by RP-HPLC method. Estimation of Canagliflozin was carried out using Shimadzu, Prominence LC system comprising COSMICSIL $100 \mathrm{C}_{18} 5 \mu \mathrm{m}$ column at room temperature with mobile phase containing HPLC grade Acetonitrile and Water in the ratio $70: 30, \mathrm{pH}$ adjusted to 3.0 using ortho phosphoric acid. The flow rate of the mobile phase was $1.0 \mathrm{ml} / \mathrm{m}$, injection volume $20 \mu \mathrm{l}$ and detection wavelength $282 \mathrm{~nm}$. Each determination carried out in triplicate and results are given in Table $2 .{ }^{13}$

\section{Buoyancy studies}

The time taken by the in-situ gel to buoyant on the surface of the medium (floating lag time) was noted. The time duration the formulation constantly floated on the surface of the dissolution medium (duration of floating) was noted. ${ }^{13}$ (Table 2 )

\section{In-vitro drug release}

The cumulative in-vitro drug release (CDR) was determined using USP dissolution apparatus II having 900 $\mathrm{ml}$ of $0.1 \mathrm{~N}$ Hydrochloric acid of $\mathrm{pH} 1.2(0.1 \mathrm{~N} \mathrm{HCl}$ of $\mathrm{pH} 1.2$ ) with $0.75 \% \mathrm{w} / \mathrm{v}$ of sodium lauryl sulphate. The medium temperature was maintained at $37^{\circ} \mathrm{C}$. Ten $\mathrm{ml}$ of in-situ gelling formulation was placed into the petridish of $4.5 \mathrm{~cm}$ internal diameter and petridish containing formulation was kept in the dissolution vessel. Then carefully transferred the dissolution medium into the dissolution vessel without disturbing the formulation. At each time interval, a precisely measured sample of the dissolution medium was removed and replenished with prewarmed $\left(37^{\circ} \mathrm{C}\right)$ fresh medium. Amount of Canagliflozin in withdrawn samples was estimated by RP-HPLC method as mentioned in drug content determination. Interference from the excipients was negligible. Each study was conducted in triplicate till 8 h. ${ }^{13}$ (Table 3)

\section{Analysis of drug release mechanism}

Several kinetics models describe drug dissolution from immediate and modified release dosage forms. There are several models to represent the drug dissolution profiles where $\mathrm{f}$ is a function of $\mathrm{t}$ (time) related to the amount of drug dissolved from the pharmaceutical dosage system. The quantitative interpretation of the values obtained in the dissolution assay is facilitated by the usage of a generic equation that mathematically translates the dissolution curve in function of some parameters related with the pharmaceutical dosage forms. All the formulations were analysed for release kinetics by different models Zero order, First order, Higuchi's diffusion Hixson Crowell and KorsmeyerPeppas models. ${ }^{14,15}$ (Table 4)

\section{Scanning electron microscopy}

Scanning Electron Microscopic evaluation was carried out using TESCAN VEGA 3 instrument. The sample housing is cleaned with a damp wipe and a SEM substrate (stub) is prepared for receiving a filter by applying a double-sided, sticky conductive carbon pad to the stub surface. The underside of the stub is labeled with the sample number using a permanent marker. The filter is removed and placed on the stub and the stub is placed in a vacuum evaporator for coating at a vacuum of $5.0 \times 10^{-5}$ torr. The filter is then removed and placed in a clean polycarbonate storage box for transfer. When examining the sample, randomly located areas are selected for higher magnification scanning. Specific areas are scanned, morphology recorded and a spectrum is collected. Representative micrographs and spectra are stored digitally.

\section{RESULTS AND DISCUSSION}

\section{Drug identification and authentication}

As a prerequisite for any formulation preformulation determination like solubility and melting point were determined and the drug Canagliflozin was found to 
be soluble in ethyl alcohol, methanol and practically insoluble in water and $0.1 \mathrm{~N} \mathrm{HCl}$. Melting point was found to be $107.2^{\circ} \mathrm{C}$ which was further confirmed by DSC. FTIR spectral data showed a characteristic conjugated low $\mathrm{C}=\mathrm{O}$ peak around $1500 \mathrm{~cm}^{-1}$ and free, intermolecular alcohols (-OH) from $3358 \mathrm{~cm}^{-1} 3738$ $\mathrm{cm}^{-1}$ and $\mathrm{C}=\mathrm{O}$ stretch at $1743 \mathrm{~cm}^{-1}$. All these findings were matched with literature values and found concurrent.

\section{Drug polymer compatibility studies}

The results obtained from preformulation compatibility studies (Figure 1 and 2) revealed that all the major peaks for drug and polymer were retained in FTIR spectrum and there were no significant changes in melting point of the drug (around $109^{\circ} \mathrm{C}$ ) in physical mixture as seen in DSC thermographs. The results infer that the drug Canagliflozin is compatible with polymer.

\section{Rheological properties}

Flow property of liquid formulations is the major concern for pour ability and swallowing. Hence viscosities of the formulations were determined at 50, 75, $100 \mathrm{rpm}$ to get correspondent angular velocities and tabulated in Table 2. The viscosity of the formulations increased with an increase in sodium alginate concentration from 0.5 to $1.5 \% \mathrm{w} / \mathrm{v}$. This phenomenon is as a consequence of increasing chain interaction with increase in polymer concentration. The calcium carbonate, which is the source of cation, when added decreased the viscosity of the formulation.

\section{Buoyancy studies}

The ideal gastroretentive system must have least floating lag time. The floating lag time of all the formulations were determined and values ranges from $157 \mathrm{~s}$ to 541 s. Formulation SF6 has the least floating lag time which consists of $1 \%$ sodium alginate and $1.5 \%$ calcium carbonate. The floating lag time of SF4 and SF5 were $286 \mathrm{~s}$ and $191 \mathrm{~s}$ respectively. Increase in floating lag time could be due to less concentration of calcium carbonate compared to SF6. This could be due to the volume of gas generated was high in the formulation SF6 and also the increase in concentration of cations in the formulation facilitates the fast gelation which is responsible for less floating lag time. Floating duration of all the formula-

\begin{tabular}{|c|c|c|c|c|}
\hline \multicolumn{5}{|c|}{ Table 1: Formulation Table. } \\
SI. No. & Formulation Code & $\begin{array}{c}\text { Polymer Concentration } \\
(\% \mathbf{\%} / \mathbf{)}\end{array}$ & $\begin{array}{c}\text { Calcium Carbonate } \\
(\% w / v)\end{array}$ & $\begin{array}{c}\text { Canagliflozin } \\
(\% w / v)\end{array}$ \\
\hline 1 & SF1 & 1.5 & 0.5 & 1.0 \\
\hline 2 & SF2 & 1.5 & 1.0 & 1.0 \\
\hline 3 & SF3 & 1.5 & 1.5 & 1.0 \\
\hline 4 & SF4 & 1.0 & 0.5 & 1.0 \\
\hline 5 & SF5 & 1.0 & 1.0 & 1.0 \\
\hline 6 & SF6 & 1.0 & 1.5 & 1.0 \\
\hline 7 & SF7 & 0.5 & 0.5 & 1.0 \\
\hline 8 & SF8 & 0.5 & 1.0 & 1.0 \\
\hline 9 & SF9 & 0.5 & 1.5 & 1.0 \\
\hline
\end{tabular}

\begin{tabular}{|c|c|c|c|c|c|c|}
\hline \multirow{2}{*}{ SI. No. } & \multirow{2}{*}{ Formulation } & \multicolumn{3}{|c|}{ Viscosity at rpm in $\mathrm{mPs}$} & \multirow{2}{*}{$\begin{array}{l}\text { Drug Content }(\%) \pm S D \\
(n=3)\end{array}$} & \multirow{2}{*}{$\begin{array}{c}\text { FLT in Seconds } \pm \text { SD } \\
(n=3)\end{array}$} \\
\hline & & 50 & 75 & 100 & & \\
\hline 1 & SF1 & 1009 & 991 & 754 & $100.47 \pm 0.15$ & $270.00 \pm 8.69$ \\
\hline 2 & SF2 & 1000 & 886 & 689 & $101.71 \pm 0.35$ & $223.83 \pm 6.01$ \\
\hline 3 & SF3 & 1102 & 1000 & 754 & $98.98 \pm 0.26$ & $173.83 \pm 6.85$ \\
\hline 4 & SF4 & 947 & 824 & 682 & $100.39 \pm 0.43$ & $286.50 \pm 3.78$ \\
\hline 5 & SF5 & 884 & 752 & 625 & $99.61 \pm 0.26$ & $191.00 \pm 3.16$ \\
\hline 6 & SF6 & 758 & 627 & 524 & $101.02 \pm 2.35$ & $157.00 \pm 5.44$ \\
\hline 7 & SF7 & 326 & 244 & 208 & $99.13 \pm 0.07$ & $259.17 \pm 7.41$ \\
\hline 8 & SF8 & 812 & 441 & 379 & $99.84 \pm 0.40$ & $421.17 \pm 29.41$ \\
\hline 9 & SF9 & 326 & 241 & 102 & $99.92 \pm 0.50$ & $541.83 \pm 17.23$ \\
\hline
\end{tabular}




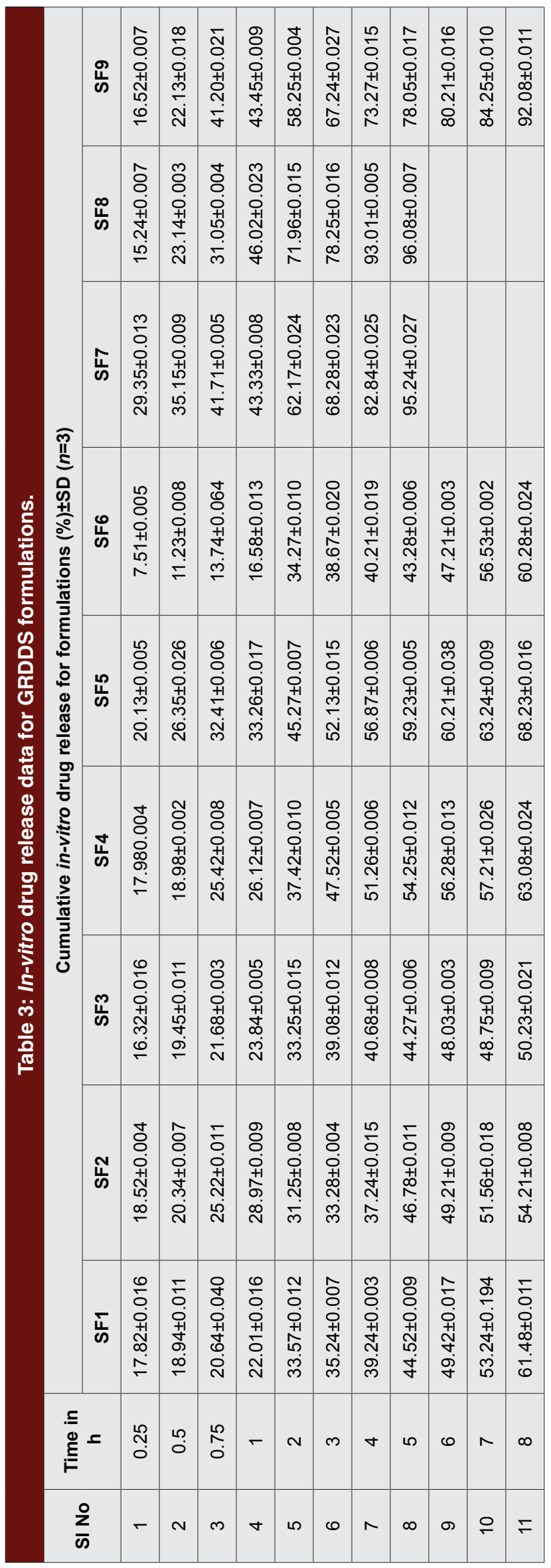

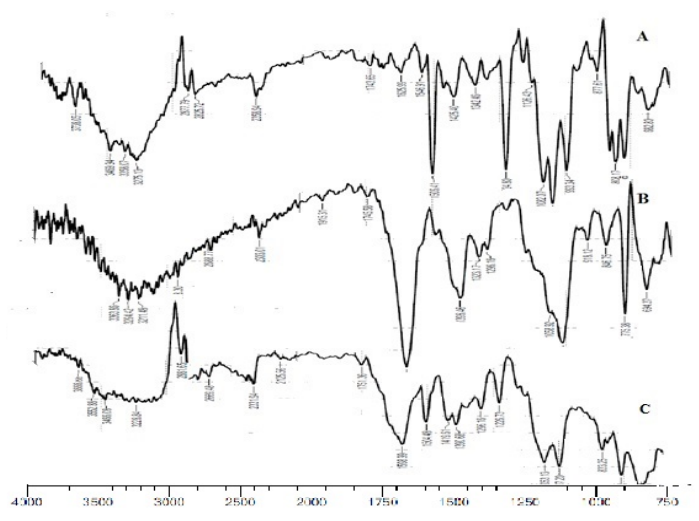

Figure 1: FTIR spectra for Canagliflozin Hemihydrate (A), Sodium Alginate (B) and Physical mixture (C).

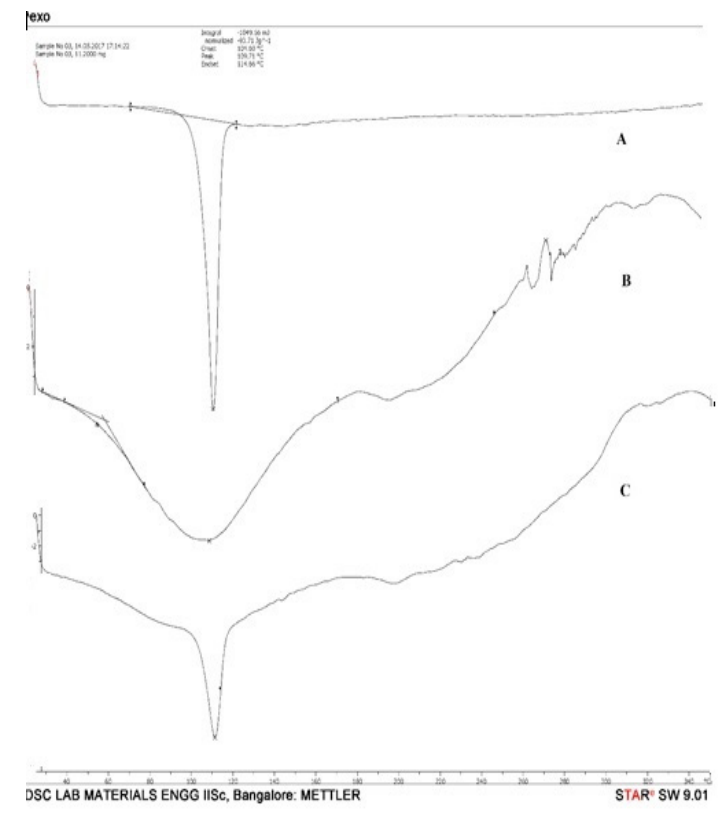

Figure 2: DSC thermogram for Canagliflozin Hemihydrate (A), Sodium Alginate (B) and Physical Mixture (C).

tions were more than $24 \mathrm{~h}$ in all the concentrations of sodium alginate and calcium carbonate.

\section{In-vitro drug release study}

All the formulations were subjected to in-vitro drug release and cumulative drug release versus time plot was depicted in Figure 3. The results of drug release suggested that at $15^{\text {th }} \mathrm{m}$ the drug release from formulations SF5 was $20.13 \%$ and that for SF4 and SF6 was 17.98 and $7.51 \%$ respectively. The reduction of drug release from SF6 could be due to quick conversion of liquid into gel. This observation were supported by floating lag time of $157 \mathrm{~s}$ for SF6 when compared with floating lag time of $191 \mathrm{~s}$ and $286 \mathrm{~s}$ for SF4 and SF5 respectively, where the amount of drug release from these two formulations comparatively higher than SF6 and floating lag time more than SF6. This observation also sug- 


\begin{tabular}{|c|c|c|c|c|c|c|c|}
\hline \multirow{2}{*}{$\begin{array}{l}\text { SI. } \\
\text { No. }\end{array}$} & \multirow{2}{*}{$\begin{array}{c}\text { Formulation } \\
\text { Code }\end{array}$} & \multirow{2}{*}{$\begin{array}{c}\text { Zero order } \\
R^{2}\end{array}$} & \multirow{2}{*}{$\begin{array}{c}\text { First Order } \\
R^{2}\end{array}$} & \multirow{2}{*}{$\begin{array}{c}\text { Higuchi } \\
R^{2}\end{array}$} & \multirow{2}{*}{$\begin{array}{c}\text { Hixson } \\
R^{2}\end{array}$} & \multicolumn{2}{|c|}{ Korsemeyer-Peppa } \\
\hline & & & & & & $R^{2}$ & $n$-value \\
\hline 1 & SF1 & 0.9102 & 0.9424 & 0.9958 & 0.9751 & 0.9874 & 0.4801 \\
\hline 2 & SF2 & 0.8134 & 0.8757 & 0.9740 & 0.8558 & 0.9758 & 0.3669 \\
\hline 3 & SF3 & 0.8001 & 0.9457 & 0.9046 & 0.9733 & 0.991 & 0.3490 \\
\hline 4 & SF4 & 0.8618 & 0.9198 & 0.9863 & 0.9021 & 0.9705 & 0.4876 \\
\hline 5 & SF5 & 0.8083 & 0.9578 & 0.9688 & 0.9371 & 0.9468 & 0.4166 \\
\hline 6 & SF6 & 0.8078 & 0.8735 & 0.9530 & 0.8532 & 0.948 & 0.4045 \\
\hline 7 & SF7 & 0.8385 & 0.9104 & 0.9829 & 0.8882 & 0.9776 & 0.4066 \\
\hline 8 & SF8 & 0.8454 & 0.8924 & 0.964 & 0.8772 & 0.9562 & 0.4200 \\
\hline 9 & SF9 & 0.7809 & 0.8709 & 0.9643 & 0.8426 & 0.9876 & 0.3350 \\
\hline
\end{tabular}

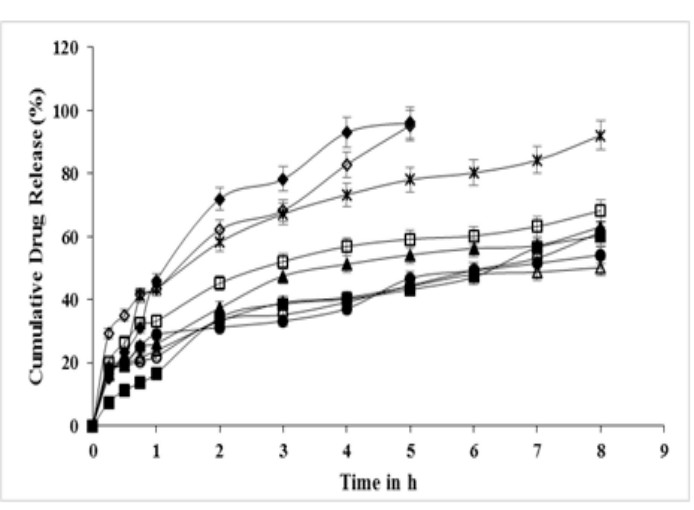

Figure 3: In-vitro drug release for formulations $\mathrm{SF}^{\circ}, \mathrm{SF}^{\bullet}$, $\mathrm{SF}^{\triangle}{ }^{\triangle}, \mathrm{SF}^{\star}{ }^{\wedge}, \mathrm{SF}^{\square}, \mathrm{SF}^{\square}, \mathrm{SF}^{\diamond}{ }^{\diamond} \mathrm{SF}^{\star}$ and SF9*.

gested that formation of gel was taken more time than SF6. The same trend continued for these formulations up to $8 \mathrm{~h}$ of drug release. Scanning electronic micrographs of gel formed at $3^{\text {rd }} \mathrm{h}$ and $5^{\text {th }} \mathrm{h}$ from SF5 formulation were studied and revealed that the formation of highly porous gel (Figure 4). This could be the reason for higher amount of drug release from SF5. The same pattern of drug release was found in SF1, SF2 and $\mathrm{SF} 3$. Among the formulations $\mathrm{SF} 7, \mathrm{SF} 8$ and $\mathrm{SF} 9$, in $\mathrm{SF} 7$ the amount of drug release at $15^{\text {th }} \mathrm{m}$ was $29.35 \%$, SF8 $15.24 \%$ and SF9 $16.53 \%$. The increase in amount of drug release from SF7 suggested that the concentration of calcium carbonate was not enough to convert liquid form to gel and it forms a highly porous gel through which the drug was released. In the concentration of $1 \%$ and $1.5 \%$ of calcium carbonate stiff gel was formed there by it reduced the amount of drug release at $15^{\text {th }}$ $\mathrm{m}$. This observation was supported by floating lag times 259 s, 421 s and 547 s for SF7, SF8 and SF9 respectively. All these results of drug release and floating lag time of all the formulations suggested the influence of calcium carbonate on gelling properties of sodium alginate.

These findings were correlated with the software assisted analysis of the responses (FLT, Viscosity, \% in-vitro drug release at $1 \mathrm{~h}$ and $8 \mathrm{~h}$ ) with respect to the

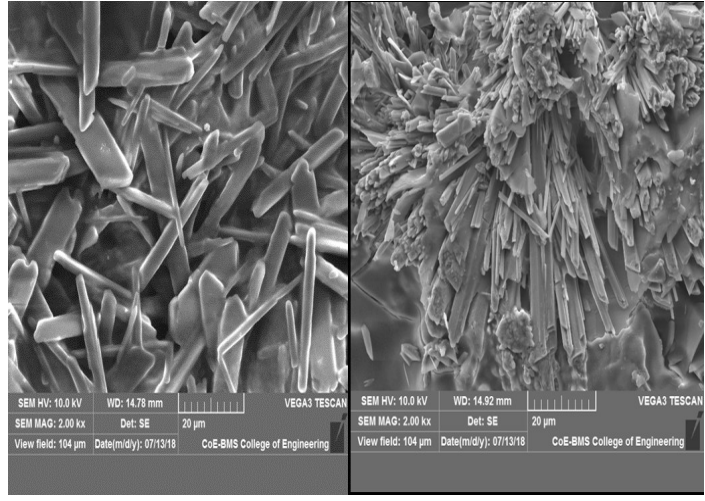

Figure 4: Scanning Electron Microscopic micrograms of SF5 formulation at $3 \mathrm{~h}$ and $5 \mathrm{~h}$ dissolution given in $2.00 \mathrm{kx}$ magnification.

two different factors namely polymer concentration (A) and calcium carbonate concentration (B) at 3 different levels. $(-1,0,+1)$ (Table 5). Fit analysis, ANOVA and surface response 3D plots (Figure 5) for different responses were studied in detail. Fit analysis suggested linear model irrespective of response factors. Equations given as follows represent the effects of each factor on corresponding response, where A denotes sodium alginate concentration and $\mathrm{B}$ denotes calcium carbonate concentration.

Floating Lag Time

$=+280.48-92.42 \mathrm{~A}$ $+9.50 \mathrm{~B}$

Viscosity

A -31.83 B

Drug Release at $1 \mathrm{~h}$ $-1.34 \mathrm{~B}$

Drug Release at $8 \mathrm{~h}$ $-3.21 \mathrm{~B}$$$
=+667.33+325.17
$$$$
=+30.50-9.73 \mathrm{~A}
$$$$
=+70.04-19.75 \mathrm{~A}
$$

For all the responses the model suggested is the linear model, ANOVA results were significant with $p$-values less than 0.05 . The predicted and adjusted $R^{2}$ values are in reasonable agreement with each other. Adequate precision, i.e., signal to noise ratio is greater than 4 which is desirable. After setting the floating lag time, viscosity of the formulation to minimum possible values, release at 1 


\begin{tabular}{|c|c|c|c|c|c|c|c|c|c|}
\hline \multirow[b]{2}{*}{ Std } & \multirow[b]{2}{*}{ Run } & Polymer & Gas generating agent & FLT in & Viscosity & $\%$ CDR & $\%$ CDR & $\%$ CDR & $\%$ CDR \\
\hline & & $\begin{array}{c}\text { Conentation } \\
(\%)(\mathrm{X} 1)\end{array}$ & Concentration $(\%)(\mathrm{X} 2)$ & Seconds (Y1) & $\begin{array}{l}\text { in } \mathrm{mPs} \\
(\mathrm{Y} 2)\end{array}$ & $\begin{array}{l}\text { at } 1 \mathrm{~h} \\
\text { (Y3) }\end{array}$ & at $3 \mathrm{~h}$ & at $5 \mathrm{~h}$ & $\begin{array}{c}\text { at } 8 \mathrm{~h} \\
(\mathrm{Y} 4)\end{array}$ \\
\hline 6 & 1 & 1.5 & 1 & 223.83 & 886 & 28.97 & 33.28 & 46.78 & 54.21 \\
\hline 9 & 2 & 1 & 1 & 191.00 & 752 & 32.41 & 52.13 & 59.23 & 68.23 \\
\hline 8 & 3 & 1 & 1.5 & 157.00 & 627 & 16.58 & 38.67 & 43.28 & 60.28 \\
\hline 3 & 4 & 0.5 & 1.5 & 541.83 & 241 & 43.45 & 67.24 & 78.05 & 92.08 \\
\hline 5 & 5 & 0.5 & 1 & 421.16 & 441 & 46.02 & 78.25 & 95.21 & 96.08 \\
\hline 4 & 6 & 1.5 & 1.5 & 173.83 & 1000 & 22.01 & 35.24 & 44.52 & 61.48 \\
\hline 2 & 7 & 1.5 & 0.5 & 270.00 & 991 & 23.84 & 39.08 & 44.27 & 50.23 \\
\hline 1 & 8 & 0.5 & 0.5 & 259.16 & 244 & 43.33 & 68.28 & 95.24 & 95.24 \\
\hline 7 & 9 & 1 & 0.5 & 286.50 & 824 & 26.12 & 47.52 & 54.25 & 63.08 \\
\hline
\end{tabular}

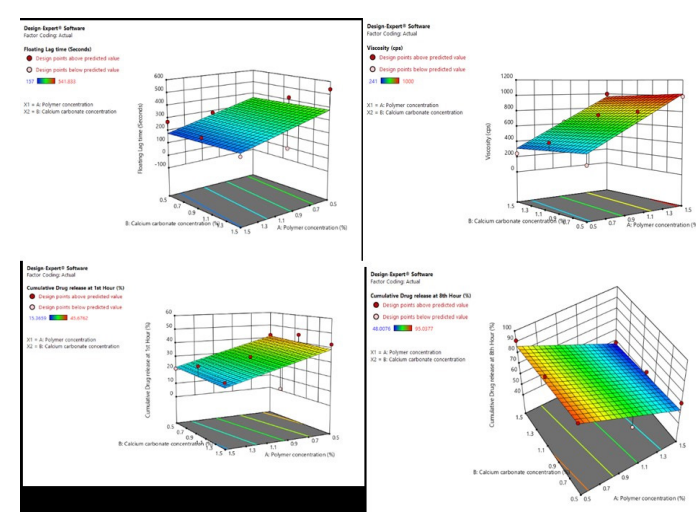

Figure 5: Three dimensional plots of formulation responses FLT, viscosity, \% cumulative drug release at $1 \mathrm{~h}$ and $8 \mathrm{~h}$ for SF5.

$\mathrm{h}$ to $30 \%$ and release at $8 \mathrm{~h}$ to $90 \%$ to attain the desired goals the search for the optimized formulation was carried out with the help of numerical optimization. The optimized formulation contained $0.868 \%$ sodium alginate and $0.957 \%$ calcium carbonate to get floating lag time of $304.03 \mathrm{~s}$, viscosity of $584.31 \mathrm{cps}$, drug release at $1 \mathrm{~h} 33.18 \%$ and 8 h $75.52 \%$ respectively suggested by software. Considering the concentration of sodium alginate and calcium carbonate by the software was nearly matched with SF5 formulation. Graphical optimization chart with design space showing the optimized region with optimized formula is depicted in Figure 6.

\section{Mechanism of drug release}

Canagliflozin release from the formulations showed linearity towards Higuchi's square root model, indicating the release mechanism to be diffusion based. $R^{2}$ values varied from 0.9046 for SF3 to 0.9958 for SF1. Results were tabulated in Table 4 . The release exponents $(n)$ of the formulations from Korsemeyer-Peppa model suggested that depending upon the formulation variables
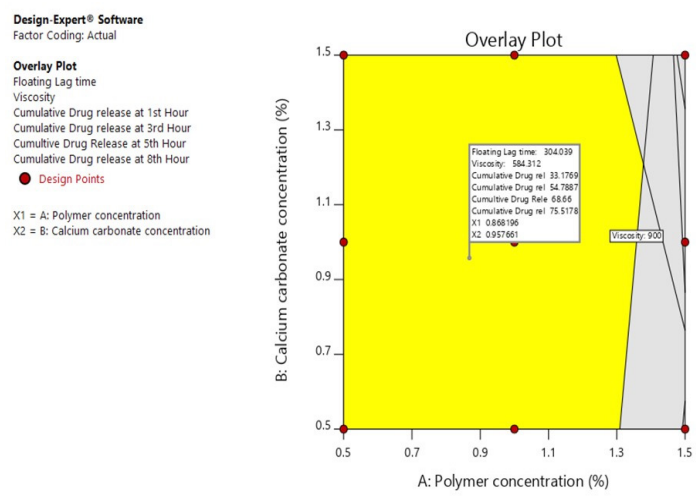

Figure 6: Graphical representation of optimization with flag stating optimized formulation parameters.

the Canagliflozin followed Fickian mechanism $(n=0.5$ means Fickian diffusion, $0.5>n<1.0$ non-Fickian diffusion and $n=1.0$ Case II diffusion). $n$-values varied from 0.3350 for SF9 to 0.4876 for SF4 $(n<0.5)$. This could be attributed to the swelling behavior of the polymer in dissolution medium creating a barrier through which the drug diffuses as the time progresses.

\section{CONCLUSION}

In the present study by considering the effect of calcium cations on the gelling properties of various concentration of sodium alginate, it was concluded that the effective gelling was achieved in the formulation comprised of $1 \%$ sodium alginate with $0.5 \%, 1.0 \%$ and 1.5 $\%$ of calcium carbonate. The software also suggested the optimized formulation must consists of $0.868 \%$ of sodium alginate and $0.957 \%$ of calcium carbonate and these compositions are much near to the composition of SF5 and thus SF5 was considered as optimized formulation. 


\section{ACKNOWLEDGEMENT}

Authors are thankful to Anthem Bio Sciences Private Limited, Bengaluru, India for providing sodium alginate as a gift sample.

\section{CONFLICT OF INTEREST}

The author declare no conflict of interest.

\section{ABBREVIATIONS}

GRT: Gastric Retention Time; GET: Gastric Emptying Time; GRDDS: Gastro Retentive Drug Delivery Systems; FDDS: Floating Drug Delivery Systems; HBS: Hydrodynamically Balanced Systems; GI: Gastro Intestinal; FTIR: Fourier-Transform Infra-Red spectroscopy; DSC: Differential Scanning Calorimetry; RPM: Rotations Per Minute; RP-HPLC: Reverse Phase High Performance Liquid Chromatography; CDR: Cumulative Drug Release; SEM: Scanning Electronic Microscopy; ANOVA: Analysis of Variance.

\section{REFERENCES}

1. Singh BN, Kim KH. Floating drug delivery systems: an approach to oral controlled drug delivery via gastric retention. J Controlled Release. 2000;63(3):235-59.

2. Bardonnetb PL, Faivre V, Pugh WJ, Piffaretti JC, Falson F. Gastroretentive dosage forms: Overview and special case of Helicobacter pylori. J Controlled Release. 2006;111(1-2):1-18.
3. Yang L, Eshraghi J, Fassihi R. A new intragastric delivery system for the treatment of Helicobacter pylori associated gastric ulcer: in vitro evaluation. J Controlled Release. 1999;57(3):215-22.

4. Chavanpatil MD, Jain P, Chaudhari S, Shear R, Vavia PR. Novel sustained release, swellable and bioadhesive gastroretentive drug delivery system for ofloxacin. Int J Pharm. 2006;316(1-2):86-92.

5. Jain SK, Agrawal GP, Jain NK. A novel calcium silicate based microspheres of repaglinide: in vivo investigations. J Controlled Release. 2006;113(2):111-6.

6. Joseph NJ, Lakshmi S, Jayakrishnan A. A floating-type oral dosage form for piroxicam based on hollow polycarbonate microspheres: in vitro and in vivo evaluation in rabbits. J Controlled Release. 2002;79(1-3):71-9.

7. Ramesh CN, Srinatha A, Jayanta KP. In situ Forming Formulation: Development, Evaluation and Optimization Using $3^{3}$ Factorial Design. AAPS Pharm Sci Tech. 2009;10(3):977-84.

8. Sheila SB, Olga H. Canaglifl ozin (Invokana), a Novel Oral Agent For Type-2 Diabetes. Drug Forecast. 2013;38(11):656-66.

9. Qin C, Wu M, Xu S, Wang X, Shi W, Dong $Y$, et al. Design and optimization of gastro-floating sustained-release tablet of pregabalin: in-vitro and in-vivo evaluation. Int J Pharm. 2018;545(1-2):37-44.

10. Rajani S, Panna T, Ranendra N S. In-vitro and in-vivo evaluation of gastroretentive floating drug delivery system of ofloxacin. Asian J Pharm Sci. 2013;8(3):191-8.

11. Sheshank S, Bhumika M, Sunil K, Vikas R. A QbD approach for the fabrication of immediate and prolong buoyant cinnarizine tablet using polyacrylamide-gcorn fibre gum. Int J Bio Macromol. 2018;117:350-61.

12. Singh B, Kaur A, Dhiman S, Garg B, Khurana RK, Beg S. QbD-Enabled Development of Novel Stimuli-Responsive Gastroretentive Systems of Acyclovir for Improved Patient Compliance and Biopharmaceutical Performance. AAPS PharmSciTech. 2016;17(2):454-65.

13. Rohith G, Sridhar B K, Srinatha A. Floating drug delivery of a locally acting $\mathrm{H}_{2}$-antagonist: An approach using an in-situ gelling liquid formulation. Acta Pharm. 2009;59(3):345-54.

14. Raghavendra NN, Chakka G, Subba RD. Design expert supported mathematical optimization of repaglinide gastroretentive floating tablets: Invitro and in-vivo evaluation. Future J Pharm Sci. 2017;3(2):140-7.

15. Paulo C, Jose MSL. Modeling and comparison of dissolution profiles. Eur $\mathrm{J}$ Pharm Sci. 2001;13(2):123-33.

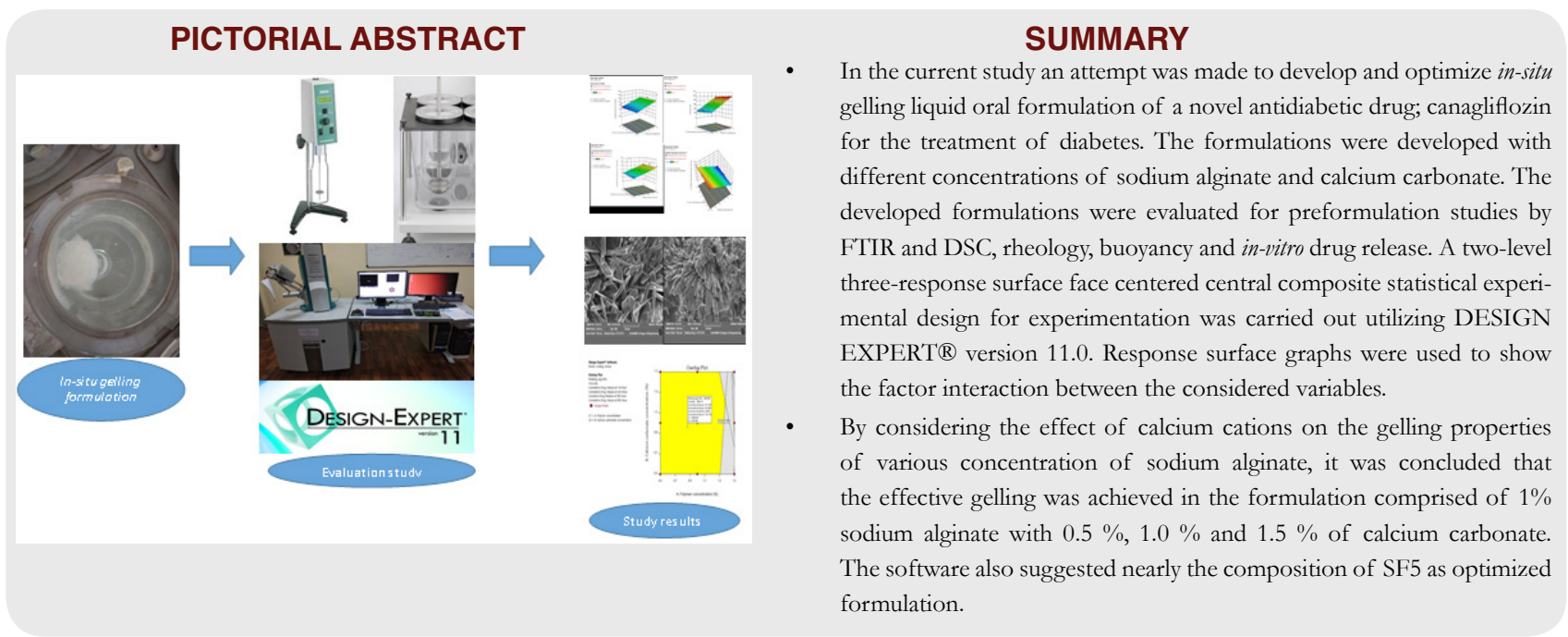

Cite this article: Rohit GB, Sadashivaiah R, Satheeshababu BK. Formulation, Optimization and Evaluation of insitu Gelling Liquid Oral Formulation of a Novel Antidiabetic Drug: Canagliflozin. Indian J of Pharmaceutical Education and Research. 2019;53(2S):s121-s128. 